\title{
In-Vitro Evaluation of Antimicrobial Activities of Escherichia coli, Klebsiella pneumoniae, Salmonella typhi, Neisseria gonorrhoeae, and Candida albicans Nosodes
}

\author{
Renuka Munshi ${ }^{1}$ Gitanjali Talele ${ }^{2}$ Rajesh Shah ${ }^{2}$ (1) \\ ${ }^{1}$ Department of Clinical Pharmacology, TNMC \& BYL Nair Ch. Hospital, \\ Mumbai, Maharashtra, India \\ ${ }^{2}$ Research Department, Life Force Foundation Trust, Mumbai, \\ Maharashtra, India \\ Address for correspondence Rajesh Shah, MD, Life Force, 411-Krushal \\ Commercial Complex, GM Road, Chembur, Mumbai 400089, \\ Maharashtra, India (e-mail: sanjivak@gmail.com).
}

Homeopathy 2022;111:42-48.

\begin{abstract}
Background This study presents the results of the minimum inhibitory concentration (MIC) assay of a series of nosodes: namely Escherichia coli, Klebsiella pneumoniae, Salmonella typhi, Neisseria gonorrhoeae, and Candida albicans. Each was tested against its corresponding infection as well as cross infections.

Methods In-vitro efficacy of polyvalent nosodes was tested using the MIC assay technique. The nosodes, namely C. albicans polyvalent nosode (35c, 100c), N. gonorrhoeae (35c), K. pneumoniae (35c, 100c), E. coli polyvalent nosode (35c, 100c) and Salmonella typhi polyvalent nosode (30c, 100c), were tested along with positive and

Keywords

- antibacterial

- nosodes

- inhibitory concentration

- potentized

- in vitro

- Escherichia coli

- Klebsiella pneumoniae

- Salmonella typhi

- Neisseria gonorrhoeae

- Candida albicans negative controls. Nosodes were studied in different potencies and at 1:1 dilution.

Results C. albicans polyvalent nosode 35c, 100c, N. gonorrhoeae 35c, and positive control amphotericin B showed inhibition of the growth of $C$. albicans species. $K$. pneumoniae $35 \mathrm{c}$, E. coli polyvalent nosode $100 \mathrm{c}$, and meropenem (positive control) showed inhibition of the growth of $K$. pneumoniae; this effect was not seen with ceftriaxone, ofloxacin and amoxicillin antibiotics. E. coli polyvalent nosode 30c in 10\% alcohol (direct and dilution 1:1) and the positive controls ciprofloxacin, ofloxacin, and amoxicillin showed inhibition of the growth of $E$. coli. The S. typhi polyvalent nosode 30c in $10 \%$ alcohol showed inhibition of growth of S. typhi.

Conclusion This study reveals that the tested nosodes exhibited antibacterial potential against the corresponding micro-organisms and against other selected organisms studied using this assay.
\end{abstract}

\section{Introduction}

The system of homeopathic medicine introduced by Samuel Hahnemann (1755-1843) is based on the Law of Similars, which suggests that any substance having a capacity of producing disease in its crude form also has the capacity to treat a similar disease if administered in a very small dose. The functioning of the homeopathic system of medicine is compa-

received

September 7, 2020

accepted after revision

January 12, 2021

published online

May 21, 2021

rable in some respects with hormesis and vaccination (though they differ in their modes of application), and also it involves sourcing the drugs from biological materials, including live and inactivated organisms, ${ }^{1}$ their isolates, or diseased materials. Professor Joseph Lux, at the University of Leipzig around the year 1820 , introduced a related concept of "isopathy", suggesting the treatment of infection by the potentized source organisms. ${ }^{2}$

(c) 2021. The Faculty of Homeopathy.

All rights reserved.

Georg Thieme Verlag KG,

Rüdigerstraße 14,

70469 Stuttgart, Germany
DOI https://doi.org/ 10.1055/s-0041-1727149. ISSN 1475-4916. 
A nosode may be used "homeopathically" based on the matching of its symptom similarity with that of the patient, as per a drug proving or pathogenesis. When used against a specific infection, however, it is often termed "isopathy", falling under the broad umbrella of homeopathy. Various nosodes, such as Psorinum, Medorrhinum, Tuberculinum and Carcinosin, are also used for their respective corresponding diseases without formally distinguishing them as "isopathic".

The category of nosodes is undergoing a revolutionary overhaul of the old drugs (Mycobacterium tuberculosis nosode, ${ }^{3}$ Cancer nosode ${ }^{4}$ ), the introduction of new remedies (HIV nosode, ${ }^{5}$ Hepatitis C nosode, ${ }^{6}$ Escherichia coli nosode ${ }^{7}$ ), as well as their efficacy studies in the laboratory (Helicobacter pylori nosode ${ }^{8}$ ), animal ${ }^{9}$ and in-vitro models (Plasmodium falciparum nosode ${ }^{10}$ ), and human studies (influenza, ${ }^{11}$ HIV, $^{12}$ and leptospirosis nosode ${ }^{13}$ ). In an animal trial, piglets in the homeopathy-treated group had significantly less $E$. coli diarrhea than piglets in the placebo group $(p<0.0001) .{ }^{14}$ In a study, no significant inhibitory effect of non-nosode drugs, such as Apis mellifica, Cantharis, Causticum hahnemanni, Staphysagria, Nux vomica, Berberis vulgaris and Lycopodium clavatum in 30c potency, was observed. ${ }^{15}$

Homeopathic nosodes sourced from any organism can be explored for its prophylactic as well as its therapeutic role in treating the disease caused by the same organism as well as in other conditions. There are over 45 common nosodes sourced from bacteria (Tuberculinum, Diphtherinum, Streptococcinum), viruses (Morbillinum [measles], Variolinum [smallpox], HIV), parasites (Psorinum [scabies]), and diseased tissues (Carcinosin). The use of Psorinum ${ }^{16}$ (sourced from a discharge containing scabies parasites) is not restricted to the treatment of scabies but may also be beneficial against cancer, ${ }^{17}$ eczema, anxiety disorders, migraines, and more. HIV nosode and Hepatitis C nosode have shown efficacy not only against HIV or Hepatitis C infections ${ }^{18}$ but also against cancer ${ }^{19}$ in pre-clinical studies. A literature search reveals that Bacillus Calmette-Guérin (BCG) was first used as immunotherapy to treat superficial bladder cancer. ${ }^{20}$ Clinical studies showed that the measles, mumps, and rubella (MMR) vaccine is safely and effectively used in the treatment of common warts. ${ }^{21,22}$ This is suggestive that the scope of a vaccine against disease is not restricted to the one it is sourced from, thereby allowing us to explore wider opportunities.

Most of the major nosodes developed during the latter part of the nineteenth century (1870 to 1900) were prepared from the non-characterized clinical samples of infected discharges or tissues (Tuberculinum, Bacillinum, Medorrhinum, Syphilinum, Diphtherinum), ${ }^{23}$ which did not have microbial categorization. As current knowledge of microbiology allows us to identify different strains of microbes, we have options to make individual nosode from specific strains, and also combine the related strains making bivalent ${ }^{5}$ or polyvalent nosodes such as Mycobacterium nosode. $^{3}$

Treatment of infections as well as antimicrobial resistance continues to be among the major medical challenges. Infectious diseases kill over 17 million people a year. ${ }^{24}$ Antibiotics, such as ciprofloxacin, erythromycin and ampicillin, may lose their effectiveness as microbes develop resistance. The use of alter- native methods to treat infections, and to increase the innate immunity of the body to fight against infections, is on the rise. Several homeopathic medicines (Aconite 30c, Arsenicum album 30c, Mercurius corrosivus 30c, Aspergillus niger) have been tested for their antibacterial activity in in-vitro studies. ${ }^{25-28}$

This study presents the results of the minimum inhibitory concentration (MIC) assay of a series of potentized (serially diluted and succussed), freshly prepared nosodes: namely E. coli, Klebsiella pneumoniae, Salmonella typhi, Neisseria gonorrhoeae and Candida albicans nosodes, tested against their respective infection as well as cross infections. The MIC assay is an in-vitro technique to study the lowest drug concentration that prevents visible micro-organism growth. The MIC can be helpful in establishing the level of resistance of a bacterial strain and can substantially affect the decision to use certain antimicrobial agents.

Different methods can be used to assess antibacterial activity. Agar disk diffusion assays are methods that estimate the antibacterial potential of a sample qualitatively with a zone of inhibition. The broth micro-dilution assay is a quantitative antibacterial susceptibility testing method that gives the percentage inhibition of bacteria in a micro-well. ${ }^{29}$

\section{Methods}

\section{Bacterial Strains}

Bacterial strains (American Type Culture Collection (ATCC)): E. coli (11775E, 25922, and 8739), Klebsiella pneumonia (BAA 2146), Salmonella enterica Typhimurium (51812), Salmonella enterica Typhimurium (13311), N. gonorrhoeae (43069), C. albicans (26790), and C. albicans (24433 and 60193) were purchased from Himedia Laboratories Pvt. Ltd., Mumbai.

\section{Chemicals}

Antimicrobial Agent Stock Solutions (positive controls) ciprofloxacin, ofloxacin, amoxicillin, fluconazole, amphotericin-B, meropenem and ceftriaxone were purchased from the hospital Pharmacy.

The following media used for the study were purchased from HiMedia Laboratories Pvt. Ltd., Mumbai.

- Nutrient agar for the bacterial strains.

- Sabouraud's agar for the fungal strains.

- Selective media.

- E. coli-Eosin Methylene Blue agar.

- Klebsiella pneumonia-MacConkey agar.

- Salmonella enterica Typhimurium-Xylose-Lysine-

Deoxycholate agar.

- N. gonorrhoeae-Thayer Martin agar.

- C. albicans-Bismuth Glycine Glucose Yeast agar.

\section{Method for Inoculum Preparation}

- The inoculum was prepared by making a direct broth suspension of isolated colonies selected from a 24 to 48 hour agar plate.

- The suspension turbidity was adjusted to achieve a value equivalent to a 0.5 McFarland turbidity standard 
Table 1 McFarland standard

\begin{tabular}{|l|l|l|l|l|}
\hline No. & $\begin{array}{l}\text { McFarland } \\
\text { standard }\end{array}$ & $\begin{array}{l}1 \% \mathrm{BaCl}_{2} \\
(\mathrm{~mL})\end{array}$ & $\begin{array}{l}1 \% \mathrm{H}_{2} \mathrm{SO}_{4} \\
(\mathrm{~mL})\end{array}$ & $\begin{array}{l}\text { Approximate } \\
\text { bacterial } \\
\text { suspension/mL }\end{array}$ \\
\hline 1. & 0.5 & 0.05 & 9.95 & $1.5 \times 10^{8}$ \\
\hline 2. & 1 & 0.1 & 9.9 & $3.0 \times 10^{8}$ \\
\hline 3. & 2 & 0.2 & 9.8 & $6.0 \times 10^{8}$ \\
\hline 4. & 3 & 0.3 & 9.7 & $9.0 \times 10^{8}$ \\
\hline 5. & 4 & 0.4 & 9.6 & $1.2 \times 10^{9}$ \\
\hline 6. & 5 & 0.5 & 9.5 & $1.5 \times 10^{9}$ \\
\hline 7. & 6 & 0.6 & 9.4 & $1.8 \times 10^{9}$ \\
\hline 8. & 7 & 0.7 & 9.3 & $2.1 \times 10^{9}$ \\
\hline 9. & 8 & 0.8 & 9.2 & $2.4 \times 10^{9}$ \\
\hline 10. & 9 & 0.9 & 9.1 & $2.7 \times 10^{9}$ \\
\hline 11. & 10 & 1.0 & 9.0 & 0 \\
\hline
\end{tabular}

(-Table 1-McFarland standard). This resulted in a suspension containing approximately $1 \times 10^{8}$ colony forming units (CFU)/mL for bacterial strains and $1 \times 10^{6} \mathrm{CFU} / \mathrm{mL}$ for fungal strains.

- The inoculum suspension was diluted in the broth such that, after inoculation, each tube contained approximately $5 \times 10^{5} \mathrm{CFU} / \mathrm{mL}$.

\section{Method for McFarland Standard Preparation}

For the preparation of $0.5 \mathrm{McF}$ arland standard, $0.5 \mathrm{~mL}$ of $1.175 \%$ $\mathrm{w} / \mathrm{v}$ barium chloride $\left(\mathrm{BaCl}_{2}\right)$ was added to $85 \mathrm{~mL}$ of $1 \%$ sulfuric acid $\left(\mathrm{H}_{2} \mathrm{SO}_{4}\right)$ and mixed. After mixing, $1 \%$ sulfuric acid was further added to make the volume up to $100 \mathrm{~mL}$. Prepared solutions were checked for its optical density at $540 \mathrm{~nm}$ range which gives absorbance ranging from 0.12 to 0.19 .

\section{Preparation of Positive Controls}

Different concentrations of positive controls (antimicrobial agents) ranging from 1 to $256 \mu \mathrm{g} / \mathrm{mL}$ were prepared.

\section{Nosodes: Test Sample Preparations}

All organisms were procured from ATCC; each strain was cultured in a cell culture laboratory. Each nosode was sourced from its respective live organism culture. A polyvalent nosode is a combination of more than two strains and preparations were made using different methods. Nosodes were potentized as per the method described in the Homoeopathic Pharmacopoeia of India (HPI). The polyvalent nosodes of S. typhi and E. coli were prepared by two methods: from the cell-free extract and the entire cells. For the MIC assays, both cell-free extract and the entire cells preparations of $S$. typhi and $E$. coli were combined, respectively.

1. S. Typhi and E. coli ${ }^{6}$ nosodes were prepared by the following methods:

\section{Method 1: Cell-Free Extract}

Twenty billion cells were sonicated using a sonicator (Citizen /CUB 2.5) until most of the bacterial cells ruptured. The material was then centrifuged at $10,000 \mathrm{rpm}$ for 30 minutes using a Hettich centrifuge. The supernatant was treated with an equal volume of strong alcohol which serves $1 \mathrm{x}$ potency. One part of $1 \mathrm{x}$ was diluted up to 100 (1:99 ratio) using alcohol (90\% v/v alcohol procured from Merck, Ethanol CAS No. 64-175, EC Number 200-578-6) for 2c potency; further potencies were prepared by serial dilution (1:99) and succussion.

\section{Method 2: Entire Cell Extract}

The nosode was prepared using 20 billion cells and not from the cell-free extract containing only endotoxin.

\section{Klebsiella pneumoniae nosode}

Twenty billion viable cells were sonicated (Citizen ultrasonic cleaner/CUB 2.5) until most of the cells ruptured. The material was treated with an equal volume of strong alcohol to make $1 \mathrm{x}$ potency. The $1 \mathrm{x}$ was diluted up to 100 (1:99 ratio) using alcohol ( $90 \% \mathrm{v} / \mathrm{v}$ alcohol, as above) for 2c potency; further potencies were prepared by serial dilution (1:99) and succussion.

3. N. gonorrhoeae and C. albicans nosodes were prepared by the trituration method

An isolated cell palate of 20 billion cells of respective organisms ( $N$. gonorrhoeae/C. albicans) was triturated with Saccharum lactis powder to make $1 \mathrm{x}$ preparation. $1 \mathrm{x}$ to $6 \mathrm{x}$ potencies were prepared by maintaining a $1: 9$ ratio. Two parts of $6 x$ were diluted up to 100 ( $1: 99$ ratio) by using $50 \%$ water for injection and $50 \%$ alcohol, and potentized to achieve $4 \mathrm{c}$ potency. As per description in the $\mathrm{HPI}^{1}$ and the Homeopathy Pharmacy textbook ${ }^{30}$ the preparation of liquid potencies is made by converting $6 \mathrm{x}$ to $4 \mathrm{c}$. Two parts of $6 \mathrm{x}$ were taken to compensate the possible material loss during the process. One part of 4c potency was diluted using 99 parts of alcohol to arrive at $5 c$ potency. Likewise, serial dilutions were done to prepare further potencies using dispensing alcohol.

All the potencies by succussion were made using an electromechanical potentizer imparting a standard and quantifiable force parameter ${ }^{31}$ (for example, 30c potency $=12,099 \mathrm{Nm}, 35 \mathrm{c}=14,115 \mathrm{Nm}, 100 \mathrm{c}=40,330 \mathrm{Nm}$ ).

E. coli polyvalent nosode (35c, 100c), K. pneumoniae (35c, 100c), S. typhi polyvalent nosode (30c, 100c), N. gonorrhoeae (35c), and C. albicans polyvalent nosode (35c, 100c) were used for the MIC experiments.

\section{Method for the MIC Assay}

- The stock solutions of the antimicrobial agent/s were prepared in the required concentrations.

- Two-fold dilutions of the antimicrobial agent/s were prepared volumetrically in the broth.

- The inoculum was prepared by making a direct broth suspension of isolated colonies selected from a 24 to 48 hour agar plate, and the adjusted inoculum suspension was diluted in the broth such that, after inoculation, each tube contained approximately $5 \times 10^{5} \mathrm{CFU} / \mathrm{mL}$.

- One milliliter of the adjusted inoculum was added to each tube containing $1 \mathrm{~mL}$ of antimicrobial agent and to the positive-control tube containing only broth and then mixed. 
- The inoculated tubes were then incubated at $37^{\circ} \mathrm{C}$ for 24 to 48 hours in an incubator.

- Growth in the tubes was checked at 24 and 48 hours.

- The amount of growth in the tubes containing the antimicrobial agent/s was compared with that observed in the positive control tubes.

- The lowest concentration at which no growth was observed in the tube was recorded as the MIC.

- For confirmation, a loop of the broth was taken from the 48 hour-old tubes and streaked on agar plates containing Nutrient agar and selective media for each organism respectively under study. The presence or absence of the growth on the agar plate was documented after 24 and 48 hours. The absence of growth indicated the inhibitory activity of medicine studied.

In-vitro efficacy of polyvalent nosode was tested using the MIC assay technique at the Department of Pharmacology, Nair Hospital, Mumbai. The polyvalent nosodes were tested along with positive and negative controls. Nosodes were studied in different potencies at 1:1 dilution. Nosodes were prepared using the vehicle $90 \%, 30 \%$ and $10 \%$ alcohol.

The nosodes prepared from each organism were tested for their antimicrobial activity against the same organism, as well as against a few other organisms (cross-activity). For example, E. coli polyvalent nosode 100c was tested against the organisms E. coli and K. pneumoniae.

\section{Results}

The MIC assay results showed that the test samples at 24 and 48 hours of incubation, E. coli polyvalent nosode 30C in $10 \%$ alcohol (direct and dilution 1:1), and the positive controls ciprofloxacin, ofloxacin and amoxicillin, could inhibit the growth of organisms of the E. coli species (-Table $2 \mathbf{A}$ ).

K. pneumoniae 35c and E. coli polyvalent nosode 100c could inhibit the growth of organisms of K. pneumoniae species when used directly. K. pneumoniae (35c, 100c), E. coli polyvalent nosode (35c, 100c), and meropenem (positive control) have shown inhibitory activity against $K$. pneumoniae species growth; however, the antibiotics (ceftriaxone, ofloxacin, and amoxicillin used as positive control) did not exhibit similar inhibitory activity (-Table 2B).

S. typhi polyvalent nosode $30 \mathrm{c}$ in $10 \%$ alcohol (direct and dilution 1:1) and the positive controls ciprofloxacin and ofloxacin could inhibit the growth of organisms of S. typhi species at 24 hours, but the effect was not continued further until 48 hours ( - Table $2 C$ ).

C. albicans polyvalent nosode (35c, 100c), N. gonorrhoeae (35c), and positive control amphotericin B could inhibit the growth of organisms of the $C$. albicans species (-Table 2D).

$N$. gonorrhoeae polyvalent nosode in $10 \%$ and $30 \%$ alcohol could not inhibit the growth of $N$. gonorrhoeae. Positive controls, ceftriaxone and ofloxacin B could inhibit the growth of $N$. gonorrhoeae organisms (-Table $\mathbf{2 E}$ ).

Photographs of the results showing the growth of $E$. coli and S. typhi are provided as - Supplementary Figs. S1 and S2, available online only.

\section{Discussion}

Evaluation of the direct microbicidal efficacy of potentized drug substances, particularly the nosodes in the cell line model, has been a relatively under-explored method in medical research. Earlier, we presumed that the homeopathic medicines would induce a therapeutic immune response only if administered to the host. Research by other scientists and by the authors has shown antimicrobial effects against malaria $^{8,9}$ and H. Pylori. ${ }^{7}$ Both possibilities call for further exploration.

Alcohol (10\%, 30\% and 90\%) as vehicle control, water, and positive and negative control were used for the comparison. Nosodes prepared in $90 \%$ alcohol were tested for their inhibitory action in a pilot study before this experiment to investigate whether $90 \%$ alcohol (vehicle) itself has microbial inhibitory activity. It was noted that for E. coli and S. typhi, respective organism growth was inhibited by $90 \%$ vehicle alcohol: hence nosodes prepared in 10 and 30\% alcohol were used as test samples.

It is interesting to note that $K$. pneumoniae (35c, 100c) and $E$. coli polyvalent nosode (35c, 100c) have shown inhibitory activity against $K$. pneumoniae species growth, even when the antibiotics (ceftriaxone, ofloxacin, amoxicillin) did not induce the inhibitory effect. Qualitatively, nosodes had shown inhibitory activity similar to the antibiotics ciprofloxacin, ofloxacin, amoxicillin, meropenem and ceftriaxone in some of the experiments, a finding that might lead to a great deal more investigative research in this area.

At the prescriber's discretion, nosodes can be used isopathically (against the specific infection) or homeopathically as per the symptom totality. The terms Nosode

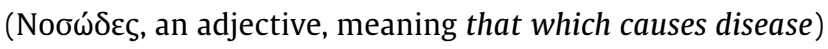
and Sarcode ( $\Sigma \alpha \rho \kappa \omega ́ \delta \varepsilon \varsigma$, meaning flesh-like) were introduced about 150 years ago before the advancement of microbiology, endocrinology and histopathology. These medicines might be considered as sarcodes (if used isopathically), but in this study the preparations have been sourced from microbes (pathogenic agents) and so they are termed here as nosodes. After having developed new drugs from the specific, characterized, strains of viruses (HIV, Hepatitis C), bacteria (M. tuberculosis, E. coli), parasites ( $P$. falciparum), fungi ( $C$. albicans), and well-defined diseased tissues (Cancer nosode ${ }^{4}$ ), the principal investigator of this project envisages a need for a developed terminology for this category of biological products, as the terms "nosode" and "sarcode" do not justify the qualitative standards of the entire new range. The scientific, professional, regulatory and pharmaceutical bodies may consider new terminology in the future.

Cross-activity was tested for those organisms that have some known organ affinity. For example, E. coli and $N$. gonorrhoeae, having urinary tract infection affinity, were examined against each other. A limitation of this study is that we have not tested the cross-activity of different nosodes against all the organisms. More studies are planned in order to validate the results. 
Table 2 MIC assay results

\begin{tabular}{|c|c|c|c|c|}
\hline No. & Name of drug tested & Conc. of drug tested & \multicolumn{2}{|l|}{ Time } \\
\hline \multicolumn{3}{|c|}{ A. Escherichia coli species } & 24 hours & 48 hours \\
\hline 1. & Ciprofloxacin & $1 \mu \mathrm{g} / \mathrm{mL}$ & - & - \\
\hline 2. & Ofloxacin & $2 \mu \mathrm{g} / \mathrm{mL}$ & - & - \\
\hline 3. & Amoxicillin & $8 \mu \mathrm{g} / \mathrm{mL}$ & - & - \\
\hline 4. & E. coli polynosode $30 \mathrm{c}$ in $10 \%$ alcohol & Direct & - & - \\
\hline 5. & E. coli polynosode $30 \mathrm{c}$ in $10 \%$ alcohol & $1: 1$ dilution & - & - \\
\hline 6. & Alcohol 10\% & Direct & + & + \\
\hline 7. & Alcohol 10\% & 1:1 dilution & + & + \\
\hline \multicolumn{3}{|c|}{ B. Klebsiella pneumoniae species } & 24 hours & 48 hours \\
\hline 8. & Ceftriaxone & $4-256 \mu \mathrm{g} / \mathrm{mL}$ (at different concentrations) & + & + \\
\hline 9. & Ofloxacin & $16-128 \mu \mathrm{g} / \mathrm{mL}$ (at different concentrations) & + & + \\
\hline 10. & Amoxicillin & $8-128 \mu \mathrm{g} / \mathrm{mL}$ (at different concentrations) & + & + \\
\hline 11. & Meropenem & $16 \mu \mathrm{g} / \mathrm{mL}$ & - & - \\
\hline 12. & K. pneumoniae $35 \mathrm{c}$ & Direct & - & - \\
\hline 13. & K. pneumoniae $100 \mathrm{c}$ & Direct & - & - \\
\hline 14. & E. coli polynosode $35 \mathrm{c}$ & Direct & - & - \\
\hline 15. & E. coli polynosode $100 \mathrm{c}$ & Direct & - & - \\
\hline 16. & Alcohol 90\% & Direct & + & + \\
\hline \multicolumn{3}{|c|}{ C. Salmonella typhi species } & 24 hours & 48 hours \\
\hline 17. & Ciprofloxacin & $1 \mu \mathrm{g} / \mathrm{mL}$ & - & - \\
\hline 18. & Ofloxacin & $2 \mu \mathrm{g} / \mathrm{mL}$ & - & - \\
\hline 19. & Salmonella typhi polynosode $30 \mathrm{c}$ in $10 \%$ alcohol & Direct & - & + \\
\hline 20. & S. typhi polynosode $30 \mathrm{c}$ in $10 \%$ alcohol & $1: 1$ dilution & - & + \\
\hline 21. & Alcohol $10 \%$ & Direct & + & + \\
\hline 22. & Alcohol 10\% & 1:1 dilution & - & + \\
\hline \multicolumn{3}{|c|}{ D. Candida albicans species } & 24 hours & 48 hours \\
\hline 23. & Amphotericin B & $8 \mu \mathrm{g} / \mathrm{mL}$ & - & - \\
\hline 24. & C. albicans polynosode $35 \mathrm{c}$ & Direct & - & - \\
\hline 25. & C. albicans polynosode: $100 \mathrm{c}$ & Direct & - & - \\
\hline 26. & Neisseria gonorrhoeae $35 \mathrm{c}$ & Direct & - & - \\
\hline 27. & Alcohol 90\% & Direct & + & + \\
\hline \multicolumn{3}{|c|}{ E. Neisseria gonorrhoeae species } & 24 hours & 48 hours \\
\hline 28. & Ceftriaxone & $16 \mu \mathrm{g} / \mathrm{mL}$ & - & - \\
\hline 29. & Ofloxacin B & $16 \mu \mathrm{g} / \mathrm{mL}$ & - & - \\
\hline 30. & N. gonorrhoeae $35 \mathrm{c}$ & Direct & + & + \\
\hline 31. & N. gonorrhoeae $100 \mathrm{c}$ & Direct & + & + \\
\hline 32. & Medorrhinum 35c & Direct & + & + \\
\hline 33. & E. coli polynosode $35 c$ & Direct & + & + \\
\hline 34. & E. coli polynosode $100 \mathrm{c}$ & Direct & + & + \\
\hline 35. & Sample 1 NG 35c & Direct & + & + \\
\hline 36. & Alcohol 90\% & Direct & + & + \\
\hline 37. & Negative control & & + & + \\
\hline
\end{tabular}

Abbreviations: -, no growth; +, growth. 


\section{Conclusion}

This study reveals that the nosodes exhibited antibacterial potential against the corresponding micro-organism and against other selected organisms studied using this assay.

\section{Highlights}

- The study presents the results of the minimum inhibitory concentration (MIC) assay of a series of nosodes: namely Escherichia coli, Klebsiella pneumoniae, Salmonella typhi, Neisseria gonorrhoeae and Candida albicans.

- E. coli polynosode 100c, K. pneumoniae 35c, S. typhi polynosode 30c, N. gonorrhoeae 35c, and C. albicans polynosode $35 \mathrm{c}$ and $100 \mathrm{c}$ have shown microbial growth inhibition.

- This study reveals that the nosodes exhibited antibacterial potential against the micro-organisms tested.

\section{Supplementary material}

Supplementary File 1 Images of results showing growth of Escherichia coli.

Supplementary File 2 Images of results showing growth of Salmonella typhi.

\section{Authors' Contributions}

Renuka Munshi provided the laboratory facility and contributed to the laboratory work and manuscript writing. Gitanjali Talele contributed to the laboratory work and manuscript writing. Rajesh Shah (the principal investigator) is the inventor of the drugs; he also developed the concept, supervised the laboratory work, and did the principal manuscript writing.

\section{Conflict of Interest}

One of the authors (Rajesh Shah, the principal investigator) has a patent pending for these nosodes.

\section{Acknowledgements}

We would like to thank Nair Hospital for providing the laboratory facility. Thanks to Lefteris Tapakis for helping in our understanding of the Greek connotation of the words "nosode" and "sarcode".

\section{References}

1 Homoeopathic Pharmacopoeia of India. 1st ed. 4th Vol;New Delhi: Ministry of Health, Government of India; 1983:136-137

2 Johann Joseph Wilhelm Lux. Accessed June 15, 2019 at: http:// www.curantur.de/English/Lux/lux.html

3 Shah R, Chowdhary A, Talele G, Vaidya S, Mukerjee S, Joshi S. Preparation, standardization, and in-vitro safety testing of Mycobacterium nosodes (Emtact polyvalent nosode). Homeopathy 2016;131:1-8

4 Shah R, Talele G. Preparation of cancer nosodes from specific cancer tissues. Int J High Dilution Res 2019;18:28-34
5 Shah R. HIV nosode: the homeopathic pathogenetic trial. Forsch Komplement Med 2015;22:156-162

6 Shah R. Clinical trial for the evaluation of a Hep C nosode in the treatment for hepatitis $\mathrm{C}(\mathrm{HCV})$ positive participants. Homeopathy 2013;102:207-214

7 Munshi R, Talele G, Shah R. Preparation and standardization of Escherichia coli nosodes sourced from select E. coli strains. Homeopathy 2020;109:207-212

8 Gosavia TP, Ghosh P, Kandhare AD, Kumar S, Subhash RR, Bodhankar L. Therapeutic effect of $H$. pylori nosode, a homeopathic preparation in the healing of chronic $H$. pylori-infected ulcers in laboratory animals. Asian Pac J Trop Dis 2012;2:S603-S611

9 Bagai U, Walter NS. Antiplasmodial potential of homeopathic drugs Chelidonium and nosode against Plasmodium berghei infection. J Complement Integr Med 2014;11:195-201

10 Joshi S, Munshi R, Talele G, Shah R. An experimental in-vitro study to evaluate the antimalarial activity of select homeopathy preparations. Int J Med Health Res 2017;7:65-68

11 Siqueira CM, Costa B, Amorim AM, et al. H3N2 homeopathic influenza virus solution modifies cellular and biochemical aspects of MDCK and J774G8 cell lines. Homeopathy 2013;102:31-40

12 Shah R. Clinical trial for the evaluation of a human immunodeficiency virus nosode in the treatment for human immunodeficiency virus-infected individuals. Indian J Res Homoeopathy. 2015;9:25-33

13 Bracho G, Varela E, Fernández R, et al. Large-scale application of highly-diluted bacteria for leptospirosis epidemic control. Homeopathy 2010;99:156-166

14 Camerlink I, Ellinger L, Bakker EJ, Lantinga EA. Homeopathy as replacement to antibiotics in the case of Escherichia coli diarrhoea in neonatal piglets. Homeopathy 2010;99:57-62

15 Pannek J, Kurmann C, Imbach E, Amsler F, Pannek-Rademacher S. In-vitro effects of homeopathic drugs on cultured Escherichia coli. Homeopathy 2018;107:150-154

16 Boericke W. Pocket Manual of Homeopathic Materia Medica and Repertory. New Delhi: B Jain Publishers; 1996:13-657

17 Mondal J, Samadder A, Khuda-Bukhsh AR. Psorinum 6x triggers apoptosis signals in human lung cancer cells. J Integr Med 2016; $14: 143-153$

18 Khuda-Bukhsh AR, Mondal J, Shah R. Therapeutic potential of HIV nosode 30c as evaluated in A549 lung cancer cells. Homeopathy 2017; 106:203-213

19 Shah R. Evaluating the anticancer effects of high-dilution preparations of carcinogens, such as HIV, hepatitis C virus, ethanol, and cancer tissues, in in-vitro models. Int J High Dilution Res. 2019;18:12-27

20 Fuge O, Vasdev N, Allchorne P, Green JS. Immunotherapy for bladder cancer. Res Rep Urol 2015;7:65-79

21 Awal G, Kaur S. Therapeutic outcome of intralesional immunotherapy in cutaneous warts using the mumps, measles, and rubella vaccine: a randomized, placebo-controlled trial. J Clin Aesthet Dermatol 2018;11:15-20

22 Dhope A, Madke B, Singh AL. Effect of measles mumps rubella vaccine in the treatment of common warts. Indian J Drugs Dermatol 2017;3:14-19

23 Allen HC. Materia Medica of the Nosodes. Reprinted ed. New Delhi: B. Jain Publishers; 1988

24 World Health Report. Accessed August 29, 2019 at: https://www. who.int/whr/1996/media_centre/press_release/en/

25 Mokarroma S, Shammi T. Determination of in-vitro antimicrobial activity of homeopathy medicines. Stamford J Microbiol 2019; 8:7-9

26 Almaguer-Flores A, González-Alva P. Antibacterial activity of homeopathic medications Lycopodium clavatum and Arsenicum album against periodontal bacteria. ODONTOS-Int J Dent Sci 2018;20:71-79

27 Suresh S, Ganesh L. Anti-bacterial effects of homeopathic remedies: In-vitro evaluation for treatment. Paper presented at: The European Congress for Homeopathy, Vienna; 2016 
48 Antimicrobial Activity of Nosodes Munshi et al.

28 Prajapati S, Sharma M, Kumar A, et al. Antimicrobial activity of different homoeopathic drugs and their potencies against 'Aspergillus niger' in vitro. Indian J Res Homoeopathy 2019; 13:150-158

29 Balouiri M, Sadiki M, Ibnsouda SK. Methods for in vitro evaluating antimicrobial activity: a review. J Pharm Analysis 2016;6:71-79
30 Banerjee DD. A Textbook of Homoeopathic Pharmacy Including Pharmacological Principles for Homoeopathic Practice. New Delhi: B. Jain Publishers; 1991:67

31 Shah R. Standardization of the potentizing machine and quantification of impact of potentization. Indian J Res Homoeopathy 2016;10:126-132 